\title{
Quale assestamento?
}

\author{
Roberto Del Favero
}

Dipartimento Territorio e Sistemi Agro-Forestali, Università di Padova, v.le dell'Università 16, I-35020 Legnaro (PD Italy) - Email: roberto.delfavero@unipd.it

\begin{abstract}
What kind of forest planning? The relationships between forest planning and silviculture are discussed, as they appear to be realized in the real world of forest management in Italy.
\end{abstract}

Keywords: Forest, Planning, Silviculture, Management, Italy

Citation: Del Favero R, 2010. Quale assestamento?. Forest@ 7: 5-6 [online: 2010-02-11] URL: http://www.sisef.it/forest@/.

Tre anni fa, in un editoriale su questa stessa rivista (Del Favero 2006) mi domandavo quale selvicoltura potesse configurarsi in Italia per i prossimi anni. Alcune delle considerazioni fatte in quell'occasione si possono considerare ancora attuali, mentre altre non hanno retto l'usura del tempo, a causa soprattutto del sopraggiungere della crisi economica mondiale e delle variazioni dei prezzi dei combustibili fossili. Ciò a dimostrare quanto sia difficile oggi ipotizzare scenari futuri o anche solo possibili evoluzioni delle attività umane.

Ciò nonostante un nuovo quesito mi è sorto come conseguenza dell'attuale dibattito sulla selvicoltura, sistemica o naturalistica, dibattito all'interno del quale non mi schiero, considerandomi un "liberalista pragmatico", convinto che, operativamente, poco diverso sarebbe il modo di operare di chi è fautore dell'una o dell'altra posizione. Tuttavia, nel giusto dibattito concettuale-filosofico fra le due concezioni emerge spesso una commistione, forse una confusione, fra selvicoltura e assestamento, cosicché parlando dell'una s'intende l'altro e viceversa. Ma allora l'assestamento e la selvicoltura coincidono o differiscono? E, in quest'ultimo caso quale assestamento va configurandosi per il futuro?

Rischiando di essere pedante, forse non è male ricordare, in estrema sintesi, che l'assestamento forestale nacque alcuni secoli fa, in centro Europa, con lo scopo di organizzare l'utilizzazione dei boschi in modo che il rifornimento di legno all'industria siderurgica non corresse il pericolo d'esaurirsi, garantendo così continuità a uno dei pilastri dell'economia dell'epoca.

L'obiettivo della costanza del legno utilizzato (ri- presa) fu poi rielaborato e arricchito di contenuti andando a configurarsi nel concetto più ampio di durevolezza. Quest'ultima, ancora in estrema sintesi, mira alla conservazione della risorsa forestale, cosicché anche le future generazioni possano goderne almeno quanto le attuali.

All'assestamento, quindi, verrebbe attribuito uno specifico compito diverso da quello proprio della selvicoltura. In altre parole, e ricorrendo per evitare "impantanamenti" ideologici ad altri settori, si potrebbe sostenere che l'assestamento starebbe alla selvicoltura come l'urbanistica all'architettura e ancora, passando al settore Sanitario, ahimè a tutti noto, il selvicoltore potrebbe essere paragonato a un primario di un reparto ospedaliero, mentre l'assestatore verrebbe personificato dal direttore sanitario, ovvero da colui che organizza logisticamente la struttura ospedaliera e ne controlla il buon funzionamento. Se si accetta questo paragone diviene calzante per l'assestamento la definizione di "organizzazione nel tempo e nello spazio delle attività selvicolturali".

Diverse, quindi, sarebbero le finalità delle due discipline: la selvicoltura quella di coltivare il bosco, l'assestamento quella di organizzare il lavoro e di controllare che l'agire del selvicoltore non abbia pregiudicato il principio della durevolezza.

Il selvicoltore, così, dovrà aver chiaro lo scopo del suo agire e conoscere le tecniche colturali adatte a meglio soddisfare gli obiettivi che si pone con la coltura. L'assestatore dovrà, invece, avere un'idea del modo ottimale di organizzare il lavoro nel rispetto della durevolezza, cioè dovrà disporre di un modello, più o meno formalmente espresso. Alla selvicoltura, quindi, la dimensione tattica e quella tecnica, 
all'assestamento quella strategica e pianificatoria.

Le differenze finora evidenziate fra assestamento e selvicoltura potrebbero, in realtà, essere solo apparenti. Infatti, almeno in Italia, l'assestatore, ovvero colui che redige il piano d'assestamento, indica anche il modo con cui il selvicoltore deve operare. Di conseguenza, nel redattore del piano confluirebbero le due figure, del selvicoltore e dell'assestatore, confermando così la commistione/confusione fra le due discipline, di cui si è detto in precedenza.

In realtà, quest'identità può esistere o meno secondo come sono intese le indicazioni selvicolturali contenute nel piano. Queste, possono essere considerate come delle ipotesi di lavoro, non vincolanti e da verificare concretamente nel momento della realizzazione dell'intervento, oppure come delle rigide prescrizioni. I due modi d'intendere tali prescrizioni sono entrambi presenti in Italia. Là dove l'assestamento è più maturo prevale nettamente la prima, dove si stanno muovendo i primi passi prevale, invece, la seconda.

Nel primo caso, anche se apparentemente sembrerebbe il contrario, l'assestamento ne esce vincente, configurandosi come una linea guida secondo la quale il selvicoltore può muoversi essendo tranquillo di rispettare il principio della durevolezza. Nel secondo caso, l'assestamento ne esce perdente, perché troppo rigido; la conseguenza è la non operatività del piano o perché non collaudato, come avviene in alcune parti d'Italia, o perché lasciato nel dimenticatoio di un cassetto di qualche polveroso archivio o, infine, perché formalmente applicato, ma, di fatto, disatteso operando diversamente. Mi pare che non vi siano dubbi su quale delle due situazioni sia preferibile. In ogni caso, in questa situazione vi è un chiaro rapporto ancillare fra le due discipline: l'assestamento sarà ancillare alla selvicoltura se verrà dato minimo peso all'aspetto pianificatorio, mentre sarà il contrario nel caso all'assestamento sia attribuito il compito di rigido prescrittore dell'operare del selvicoltore.

Tuttavia, forse è necessario fare un ulteriore passo avanti, per uscire da questa logica d'ancillarità. An- cora un esempio di tipo sanitario può forse spiegare questi possibili nuovi orizzonti. Negli anni cinquanta, quando ero molto giovane, nel mio paese di montagna vi era un piccolo ospedale privato dove un paio di medici si occupavano di tutto, dagli interventi chirurgici alle cure più strettamente mediche, dalla pediatria alla ginecologia e, soprattutto, a far funzionare l'ospedale. Un tale modello, oggi, sarebbe impensabile. Ebbene, questo modello non è poi molto diverso da quello attuale dell'assestamento-selvicoltura in Italia. All'estero, invece, già da tempo l'assestamento non si colloca culturalmente nell'area della selvicoltura, ma in quella dell'economia e/o della pianificazione territoriale.

Nel resto d'Europa, infatti, all'assestamento sono attribuiti compiti e connotati diversi da quelli classici, ossia non solo d'organizzare nel tempo e nello spazio l'attività selvicolturale, ma di valorizzare le risorse forestali di un certo territorio. È questa la via che si sta cominciando a sperimentare anche in alcune regioni italiane. In questo caso all'assestamento forestale potrebbe essere affidato il compito di organizzare e sviluppare circa una trentina di usi e funzioni attribuibili all'area forestale.

Se così concepito l'assestamento forestale, pur mantenendo il compito classico di garantire il rispetto del principio della durevolezza, assumerebbe significati e contenuti notevolmente più ampi.

Di fronte a questa prospettiva merita, tuttavia, segnalare l'attuale crisi dei processi pianificatori, comparsa con il crollo dell'ideologia comunista e l'avanzata del neo-liberalismo economico. Se sia questa la strada giusta non sta a me dirlo, tuttavia non vorrei che, dimenticando l'assestamento e con esso il principio della durevolezza, le future generazioni possano accusare le attuali di scarsa lungimiranza nei confronti della conservazione di quell'inestimabile valore costituito dal bosco.

\section{Bibliografia}

Del Favero R (2006). Quale selvicoltura?. Forest@ 3 (1): 1-2. [online] URL: http://www.sisef.it/forest@/show.php? $\mathrm{id}=334$ 